\title{
Cultural Differences in the Persuasiveness of Evidence Types and Evidence Quality
} Jos Hornikx \& Hans Hoeken

Cultural differences in reasoning and persuasion have mainly been documented for the East-West divide. Nisbett (2003) expects such differences to be absent for Western cultures because of their shared Grecian inheritance. The results of two experiments, however, show that France and The Netherlands, both Western European countries, differ with respect to the persuasiveness of different evidence types. In Study $1(\mathrm{~N}=600)$, cultural differences occurred between the relative persuasiveness of anecdotal, statistical, causal, and expert evidence. In Study $2(\mathrm{~N}=600)$, the quality of statistical and expert evidence was manipulated. For the Dutch, but not for the French, normatively strong evidence was more persuasive than normatively weak evidence for both evidence types. Implications and possible explanations are discussed.

\section{Keywords: Argument Quality; Evidence; France; Persuasion; The Netherlands}

In current theories on persuasion, it is believed that under certain conditions the quality of the arguments in a persuasive message determines the outcome of the persuasion process (Chaiken, 1987; Petty \& Cacioppo, 1986). There is abundant empirical evidence for this belief (see, for a recent review, Park, Levine, Kingsley Westerman, Orfgen, \& Foregger, 2007). An important determinant for argument quality is the type of evidence that is included in the message to support the claim. The concept of evidence has received much attention from scholars (see for reviews,

\footnotetext{
Jos Hornikx (PhD, Radboud University Nijmegen, The Netherlands) is an Assistant Professor at the Department of Business Communication Studies at Radboud University Nijmegen (The Netherlands). Hans Hoeken (PhD, Tilburg University, The Netherlands) is a Professor at the same department. We would like to thank two anonymous reviewers, Alan Sillars, Carel Jansen, Daniel J. O'Keefe, Peter Jan Schellens, and Marianne Starren for their extensive and constructive comments on previous versions of this paper. We also thank Frank van Meurs, Brigitte Planken, and Evelyne Vos-Fruit for their help with the construction and translation of the material. This paper is based on Hornikx' dissertation. Correspondence to: Jos Hornikx, Radboud University Nijmegen, Center for Language Studies, Department of Business Communication Studies, Erasmusplein 1, PO Box 9103, 6500 HD Nijmegen, The Netherlands. Tel: +31 2436155 13; Fax: +31 2436121 77; E-mail: j.hornikx@let.ru.nl
} 
Reinard, 1988; Reynolds \& Reynolds, 2002). However, one frequently asked question has remained largely unaddressed: "Does evidence function the same way in various cultures?” (McCroskey, 1969, p. 176; Greene \& Brinn, 2003; Reynolds \& Reynolds, 2002).

In this paper, we present empirical research on this issue. First, we will discuss research that has been conducted on cultural differences in reasoning, which has largely focused on differences between Easterners and Westerners. Second, we will show that there are reasons to believe that there may be differences in the response of Westerners to different evidence types, and that the comparison between The Netherlands and France is relevant in this respect. Finally, two experiments are presented in which this comparison is made.

\section{Cultural Differences in Reasoning between East and West}

Nisbett (2003) presents a large body of evidence showing that Easterners and Westerners differ from each other in many behavioral and cognitive processes. He claims that these differences are manifestations of "two utterly different approaches to the world ... that have maintained themselves for thousands of years" (p. xx). He believes that these different approaches are the result of differences in intellectual inheritance: Whereas Westerners are heavily influenced by the philosophers from ancient Greece, Easterners are heavily influenced by Taoism. Nisbett discusses empirical evidence for such cultural differences in perception and causal inference processes, the fundamental attribution error, the focus on objects or relations, and reasoning. It is the latter domain, reasoning, that is of interest to this study.

According to Nisbett, Peng, Choi, and Norenzayan (2001, p. 293), a preference for analytic reasoning is an important heritage of the ancient Greek philosophers. Westerners tend "to focus on attributes of the object to assign it to categories, and a preference for using rules about the categories to explain and predict the object's behavior." The heritage of the ancient Chinese culture, on the other hand, is believed to be a preference for holistic reasoning, which involves "an orientation to the context or field as a whole, including attention to relationships between a focal object and the field, and a preference for explaining and predicting events on the basis of such relationships" (p. 293).

Norenzayan, Smith, Kim, and Nisbett (2002, Study III) provide evidence for the impact of such reasoning differences on the acceptability of arguments. They compared the acceptance of the following two claims: "Germans are nice people" and "Polish are nice people." Both claims were supported by the same premise, namely "All Europeans are nice people." In a pretest, it was assessed that Germans were considered to be a more typical instantiation of Europeans than Polish. This difference in typicality influenced the Easterners' evaluation: They found the typical arguments (Germans) more convincing than the atypical arguments (Polish). The Westerners, on the other hand, found the arguments equally convincing regardless of typicality. 
Nisbett and his colleagues have documented more of such differences between Easterners and Westerners in reasoning processes (see, for a review, Nisbett, 2003, chap. 7). If Nisbett's explanation for these differences holds water, then differences in reasoning within Western cultures should not exist because these cultures share the same ancient Greek cultural background. However, despite their shared cultural background, there are cultural differences between Western cultures. Hofstede (1980, 2001) distinguishes five cultural dimensions on which cultures can be compared. These are individualism-collectivism, high--low uncertainty avoidance, large-small power distance, masculinity-femininity, and long-term versus short-term orientation. For some of these dimensions, Western cultures do not differ widely from each other. Most Western cultures are positioned at the individualism extreme of the individualism-collectivism dimension and at the short-term extreme of the longterm versus short-term dimension (Hofstede 2001, pp. 215, 356). On the other dimensions, large-small power distance, high-low uncertainty avoidance, and masculinity-femininity, however, Western cultures do differ from each other. In the next section, it is reasoned that differences on one of these dimensions, largesmall power distance, may lead to differences in the persuasiveness of different evidence types. Before such predictions can be made, however, the different types of evidence have to be identified first.

\section{Evidence Types}

Persuasive messages typically contain pragmatic arguments (Schellens \& Verhoeven, 1994, pp. 109-115). A claim about the desirability of a certain behavior (e.g., physical exercise) or measure (e.g., the implementation of a comprehensive exam) is supported by arguments that refer to the desirable consequences of this behavior (e.g., improved heart condition) or measure (e.g., high starting salaries). To assess the quality of a pragmatic argument, people have to evaluate (a) the probability that the consequence will occur as a result of the behavior (e.g., the likelihood that physical exercise improves the condition of one's heart), and (b) the desirability of the consequences (e.g., a strong heart) (Feteris, 2002). Several studies have shown that it is much easier for participants to assess a consequence's desirability than to assess its probability (Areni \& Lutz, 1988; Van Enschot-Van Dijk, Hustinx, \& Hoeken, 2003). It is easier to evaluate the claim that a high starting salary after graduation is desirable than to evaluate the claim that a high starting salary will be the result of implementing a comprehensive exam.

As a result, professional communicators may be more inclined to provide additional information to support a claim about the probability of a certain consequence than to provide such information to support a claim about its desirability, the assumption being that the audience can deduce the latter for itself whereas the former task is much harder. Indeed, in two content-analytic studies of persuasive brochures, additional information to support claims about the probability of a consequence was found to be included much more frequently than additional information to support claims about the desirability of a consequence (Hornikx, 
Starren, \& Hoeken, 2003; Schellens \& de Jong, 2004). Therefore, we will focus in this study on the role of different types of additional information that are provided in support of claims about the probability of a certain consequence as a result of a certain action or measure.

A basic argument can be considered as consisting of a claim, data, and a warrant that expresses the relevance of the data for the claim put forward (Toulmin, 1958, pp. 97-98). In essence, we are interested in four types of data that can be used to support claims about a consequence's probability. Following Hoeken and Hustinx (2003), we distinguish an individual example, statistical information, causal explanations, and expert opinions. ${ }^{1}$ Hoeken and Hustinx defend their categorization by referring to the parallel between these types of data and the type of data produced by empirical research methods used to assess covariation between certain characteristics. Information on a single example is typically the result of conducting a case study, whereas statistical information on the covariation between characteristics is the result of conducting a survey. Information about the causal relation between two characteristics, on the other hand, is typically the result of an experiment, while expert opinions are of a different nature but resemble the use of literature references in introductions of research papers. A claim about the covariation or causal relation between two characteristics is supported by a reference to a source who presumably has obtained (case study, survey, or experimental) evidence on that relationship. In all four cases, the additional data used to support the claim about the covariation fall within Reynolds and Reynolds' (2002, p. 429) definition of evidence: "data (facts or opinions) presented as proof for an assertion."

The persuasiveness of different evidence types can differ. Hoeken and Hustinx (2003) reported that anecdotal evidence is less persuasive compared to statistical, causal, and expert evidence. This finding was corroborated by Allen and Preiss (1997), who conducted a meta-analysis of the results of experimental studies on the relative persuasiveness of anecdotal and statistical evidence. However, the question is whether such differences in the relative persuasiveness of different evidence types are the same between Western cultures. To address this question, the relevance of certain cultural differences to the persuasiveness of certain evidence types is discussed next.

\section{Cultural Differences between Western Cultures}

Hofstede (2001, p. 98) defines power distance on a cultural level as "The extent to which the less powerful members of institutions and organizations within a country expect and accept that power is distributed unequally." In cultures with a large power distance, such as the French or the Belgian culture, people accept and expect that some people can determine other people's behavior. In cultures with a small power distance, such as the Dutch or the Swedish culture, people accept and expect such power inequalities to a lesser degree, preferring equality relationships instead. Hofstede claims that these power inequalities are reflected, for instance, in the role and the position of teachers (p. 107). In large power distance cultures, students depend on teachers, authoritarian values are adhered to regardless of one's 
educational level, and education is teacher-centered. In small power distance cultures, on the other hand, teachers treat students as equals, authoritarian values are adhered to mainly by people with a lower education, and education is student-centered.

These differences in the perception of the teacher's position may affect the relative persuasiveness of expert evidence. Kruglanski et al. (2005) suggest that the influence of experts on recipients depends on the perceived gap between the recipients' own knowledge and that of the expert. As a gap in knowledge is accepted more easily in large versus small power distance cultures, it could be argued that expert evidence may be more persuasive in these large power distance cultures than in small power distance cultures (see, e.g., Jung \& Kellaris, 2006; Pornpitakpan, 2004). Indeed, there is some empirical evidence that the use and persuasiveness of expert evidence are related to the level of power distance in a culture. Pornpitakpan and Francis (2001) compared the responses of Thai and Canadian undergraduate students who received a message in which the strength of the arguments as well as the qualifications of the source providing the arguments were manipulated. According to Hofstede (2001, p. 87), Thailand can be characterized as a large power distance culture, whereas Canada is considered to be at the lower end of the power distance continuum. The results of the study showed that whereas the attitudes of the Canadian participants were much more determined by the strength of the arguments put forward (regardless of the source of these arguments), the attitudes of the Thai participants were more influenced by the qualifications of the source (regardless of the arguments put forward).

Pornpitakpan and Francis (2001) document cultural differences in the persuasiveness of experts along the East-West dimension. However, if the difference in power distance is the cause of these differences in persuasiveness, similar differences could be obtained when comparing Western cultures. Hofstede (2001, p. 87) has shown that Western European countries differ considerably from each other on the power distance dimension. These countries can be found at the high end of the power distance dimension (e.g., France, Belgium), in the middle (e.g., Greece, Spain), as well as at the low end (e.g., The Netherlands, Sweden). If there are cultural differences in the relative persuasiveness of evidence types, the combination of power distance and expert evidence is a promising starting point.

In this study, we compare the relative persuasiveness of different evidence types for the Dutch and the French cultures. The choice of these two cultures is based on the fact that they share the same inheritance from ancient Greece, and that they are very similar in current times with respect to their economy and political structure. On the other hand, France scores even higher than Thailand on power distance, whereas The Netherlands scores slightly lower than Canada on this dimension according to Hofstede (2001, p. 87). ${ }^{3}$ This cultural difference has been found in several replications of Hofstede's study (see, for an overview, Hofstede, 2001, pp. 91-92), and has been documented in other large-scale studies as well (e.g., Koopman et al., 1999).

Furthermore, professional communicators from both countries have different intuitions about what makes a message persuasive. De Mooij (1998, pp. 277-279) showed that the older people advise younger people in ads in a large power distance 
culture (France), and also that experts in ads are disliked in a small power distance culture (The Netherlands). Hornikx et al. (2003) conducted a content-analytic study in which they compared the use of expert evidence in French and Dutch persuasive leaflets. Expert evidence was employed more frequently in the French than in the Dutch corpus, suggesting that French writers of persuasive texts have other intuitions about the persuasiveness of expert evidence than their Dutch colleagues.

In sum, with this study, we are responding to the call for research on cultural differences in the relative persuasiveness of evidence types (Greene \& Brinn, 2003; McCroskey, 1969; Reynolds \& Reynolds, 2002). We will address this issue by comparing the French and the Dutch cultures. As such, this research supplements studies in which differences between Eastern and Western cultures have been examined. Despite the shared ancient Grecian roots of these countries, the difference between them in the level of power distance suggests that expert evidence in particular may be differentially persuasive in the two cultures. In the first study, the persuasiveness of expert evidence, as well as of anecdotal, statistical, and causal evidence was assessed for Dutch and French participants.

\section{Study 1}

Study 1 examined the persuasiveness of four types of evidence in France and The Netherlands. This study also tested whether expert evidence is more persuasive to the French than to the Dutch, as could be hypothesized on the basis of corpus research by Hornikx et al. (2003).

RQ1: Are there cultural differences in the relative persuasiveness of anecdotal, statistical, causal, and expert evidence between France and The Netherlands?

H1: Expert evidence is more persuasive to the French than to the Dutch.

\section{Method}

Material. A pretest with 50 claims was conducted to select 20 claims that were seen as moderately probable by both Dutch $(n=30)$ and French students $(n=28)$. Highly probable claims do not need evidence because they are accepted without supporting evidence; highly improbable claims are rejected regardless of the supporting evidence (cf. Edwards \& Smith, 1996). An example of a moderately probable claim is "The consumption of basil in tomato pasta sauce improves sporting performance" (see Appendix for all 20 claims).

For each of the 20 claims, a set of anecdotal, statistical, causal, and expert evidence was created (see Table 1 for examples). The statistical evidence instantiations cited studies that had a large sample size $(138,246,315$, and 429$)$, and contained a high percentage of cases in the sample for which the claim held $(71 \%, 74 \%, 77 \%$, and $82 \%)$. The relatively small sample size of 138 was included because for claims participants are unfamiliar with a study conducted with a large group of participants may be perceived as unrealistic. Expert evidence was operationalized through the titles of the expert referred to (Prof. Dr), and through the relevance of the field of 
Table 1 Two of the Claims with Four Types of Evidence

\begin{tabular}{cl}
\hline Claim (4) & $\begin{array}{l}\text { The consumption of basil in tomato pasta sauce improves sporting } \\
\text { performance. }\end{array}$ \\
Anecdotal evidence & $\begin{array}{l}\text { Since Peter Johnson from Amsterdam regularly eats tomato pasta } \\
\text { sauce with basil, his sporting performance has improved. } \\
\text { The results of a French study among } 315 \text { participants showed that the } \\
\text { sporting performance of } 74 \% \text { of them has improved after having } \\
\text { regularly eaten tomato pasta sauce with basil. } \\
\text { Basil improves the production of adrenaline, the substance that is } \\
\text { necessary for better sporting performance. }\end{array}$ \\
Causal evidence & $\begin{array}{l}\text { Professor Dr Giraud, a specialist in the field of dietetics at the } \\
\text { University of Bordeaux, underscores that the consumption of basil in } \\
\text { tomato pasta sauce improves sporting performance. }\end{array}$ \\
Claim (16) & $\begin{array}{l}\text { Wearing a tie too tightly leads to reduced sight. } \\
\text { Anecdotal evidence } \\
\text { Satrick van de Ven from Apeldoorn suffers from a reduced sight since } \\
\text { he has been wearing his ties too tightly. } \\
\text { A Dutch study among 246 people showed reduced sight for } 74 \% \text { of } \\
\text { them as a result of regularly wearing a tie too tightly. }\end{array}$ \\
Causal evidence & $\begin{array}{l}\text { Wearing a tie too tightly increases pressure on the eyeball, which } \\
\text { damages the nerves, and reduces the sight. }\end{array}$ \\
According to Prof. Dr Van Zanten from Utrecht University, an expert & in eye diseases, wearing a tie too tightly leads to reduced sight. \\
\hline
\end{tabular}

expertise to the claim under consideration. These fields were selected in a session with Dutch students $(N=17)$. Dietetics, for instance, was considered as a relevant field of expertise for the claim about basil and sporting performance. For statistical and expert evidence, there were two slightly different templates, which were used twice in the questionnaire. ${ }^{4}$ Anecdotal evidence consisted of a short sentence about a person who had experienced the consequence (e.g., good sporting performance) as a result of behavior (e.g., eating basil). Causal evidence, finally, consisted in most cases of two causal relations, which is typical of everyday explanations (Hesslow, 1988). ${ }^{5}$

The Dutch claims, evidence, and instrumentation were translated into French by native Dutch speakers, and then back translated (cf. Brislin, 1980) into Dutch by native French speakers or a translation agency. The two Dutch versions were compared, and modifications were made whenever necessary. In addition, equivalent Dutch and French first names, last names, places, and universities were selected for the evidence instantiations in order to increase equivalence further (cf. Harkness, Van de Vijver, \& Mohler, 2003).

Participants. The participants were students from universities in various cities within each country. The Dutch cities were Amsterdam ( $n=42$; two classes), Delft $(n=23)$, Groningen $(n=46)$, Nijmegen ( $n=108$; three classes), and Tilburg $(n=86$; two classes). The French cities were Angers $(n=30)$, Paris $(n=107$; two classes), Roubaix ( $n=34)$, Toulouse ( $n=26)$, and Tours ( $n=98$; two classes). Three quarters of the Dutch and French participants studied at the faculty of Humanities. The percentage of female participants was higher in France $(86.8 \%)$ than in The Netherlands $(77.4 \%), \chi^{2}(1)=8.98, p<.01$, but there was no interaction effect 
between participants' sex and the relative persuasiveness of the evidence types $(F[3,596]<1)$. On average, the Dutch participants were $20.98(S D=2.36$, range 17-30) years old, and the French participants 20.75 ( $S D=1.96$, range 17-30), $t(585.16)=1.33, p=.19$.

Instrumentation. The booklet that participants received was titled "Opinions on social issues." After brief written instructions, the participants read 16 claims with evidence and 4 claims without evidence. Each of the 20 claims (with or without evidence) was followed by a repetition of the claim, and then by a 5-point semantic differential (very improbable-very probable) on which participants rated the claims' probability. ${ }^{6}$

After giving 20 judgments, participants indicated their agreement on 5-point Likert scales with several statements geared at accounting for cultural differences in the persuasiveness of evidence types. For statistical evidence, the Preference for Numerical Information scale (PNI) was included (Viswanathan, 1993). The 8-item version of the PNI proved to be reliable (Dutch: $\alpha=.85$; French: $\alpha=.86$ ). For expert evidence, a 6-item Preference for Expert Information scale (PEI) was constructed, which measures the degree to which people appreciate information given by experts. ${ }^{7}$ A factor analysis showed that four of the six items loaded on a common dimension, and these items proved to be reliable (Dutch: $\alpha=.75$; French: $\alpha=.77$ ). Finally, the Need for Cognition scale (NFC) from Cacioppo, Petty, and Kao (1984) was included (Dutch: $\alpha=.67$; French: $\alpha=.74$ ). The NFC scale served as a check because it is related to people's sensitivity to argument quality (Petty \& Cacioppo, 1986).

Next, the perceived expertise of the four experts featured in the material was measured. For each of the experts, participants indicated on a 5-point Likert scale the degree to which they agreed that the expert had enough expertise to make a judgment about the particular claim he/she supported. The questionnaire ended with questions about participants' age, sex, nationality, and current education.

Design. Five versions of the material were constructed. All participants received the 20 claims in exactly the same order, but the distribution of the evidence types over the claims and the versions followed a balanced Latin square. In each version, four claims were supported by statistical evidence, four by anecdotal evidence, four by causal evidence, four by expert evidence, and four were not supported by evidence.

Procedure and statistical tests. The questionnaire was introduced and distributed in tutorials or lectures. Informed consent was obtained in writing from with the participants' teachers beforehand, and by informing potential participants about the researchers' background, the study, and their right not to take part in the study. After the questionnaires had been collected, the real research purpose was revealed, and participants were thanked for their cooperation. The students received no reward for their participation. The whole procedure took about 10 to 12 minutes in the smaller classes, and 13 to 15 minutes in the larger classes.

RQ1 was evaluated by means of a 2 (culture) $\times 4$ (type of evidence) analysis of variance with repeated measures, where culture was a between-subjects factor and type of evidence a within-subjects factor. Type of evidence did not have five but four levels, because the no evidence condition served as a baseline against which the effect of 
providing additional evidence was assessed for the Dutch and the French sample separately. For each individual probability rating of a claim that was supported by (anecdotal, statistical, causal, or expert) evidence, the average probability rating of that claim without evidence for that culture was subtracted. The resulting score reflects the extent to which providing evidence increased the claim's perceived probability.

In addition to these analyses by participants, analyses by stimuli were also conducted in order to test whether the effects of culture and evidence type could be generalized over the different claims. $\mathrm{H} 1$ was tested with an independent $t$-test. The within-subjects design ran the risk of a carry over effect (Elmes, Kantowitz, \& Roediger, 1992, p. 121): The participants' judgments of the evidence types in the second part of the booklet may be influenced by their judgments of the evidence types in the first part. However, the interaction between time of judgment and type of evidence, suggestive of a carry over effect, was not significant, $F(3,594)=1.03, p=.38$.

\section{Results}

First, it was tested whether there were cultural differences in the probability of the claims without evidence (since they were used as a baseline for the computation of evidence effects). Independent $t$-tests were computed to assess whether there were significant differences between the French and the Dutch participants with respect to their probability ratings of the claims presented without any supporting evidence. For 3 of the 20 comparisons, a significant difference was found. The probability ratings of the Dutch participants were higher than those of the French participants in two cases, whereas in one case, the difference was in the opposite direction. ${ }^{8}$

There was no main effect of culture, $F_{1}(1,598)=3.48, p=.06 ; F_{2}(1,19)=1.84, p=$ .19 , but there was a main effect of evidence type, $F_{1}(3,596)=34.18, p<.001, \eta^{2}=.06$; $F_{2}(3,17)=19.15, p<.001, \eta^{2}=.40$. However, this main effect was qualified by a significant interaction between culture and evidence type, $F_{1}(3,596)=5.13, p<.01$, $\eta^{2}=.01 ; F_{2}(3,17)=3.62, p<.05, \eta^{2}=.15$. Separate one-way ANOVAs revealed main effects of evidence type for the French, $F_{1}(3,292)=10.53, p<.001, \eta^{2}=.04 ; F_{2}(3$, $17)=7.54, p<.01, \eta^{2}=.21$, as well as the Dutch sample, $F_{1}(3,302)=28.79, p<.001$, $\eta^{2}=.09 ; F_{2}(3,17)=16.18, p<.001, \eta^{2}=.43$. Post hoc comparisons using Bonferroni tests revealed that for the French as well as the Dutch, anecdotal evidence yielded lower scores than the other three evidence types (see Table 2). For the French sample, these

Table 2 Persuasiveness of Evidence Types

\begin{tabular}{llllll}
\hline & \multicolumn{2}{c}{ Dutch $(n=305)$} & & \multicolumn{2}{c}{ French $(n=295)$} \\
\cline { 2 - 3 } \cline { 5 - 6 } Evidence Type & $M$ & $S D$ & & $M$ & $S D$ \\
\hline Statistical & $0.39^{\mathrm{a}^{* * *}}$ & 0.66 & & $0.20^{\mathrm{a}}$ & 0.65 \\
Expert & $0.24^{\mathrm{b}}$ & 0.61 & & $0.25^{\mathrm{a}}$ & 0.70 \\
Causal & $0.24^{\mathrm{b}^{*}}$ & 0.61 & & $0.13^{\mathrm{a}}$ & 0.72 \\
Anecdotal & $-0.03^{\mathrm{c}}$ & 0.63 & & $0.00^{\mathrm{b}}$ & 0.66 \\
\hline
\end{tabular}

Note. ${ }^{\star} p<.05,{ }^{* *} p<.001$, differences between cultures. Different superscripts in the same column refer to significant differences, alpha level of .05. 
three evidence types did not differ significantly from each other, whereas for the Dutch sample, statistical evidence yielded higher scores compared to the causal and the expert evidence (which did not differ significantly from each other).

To test H1, an independent samples $t$-test was computed. Contrary to expectations, expert evidence did not lead to higher scores for the French participants compared to those of the Dutch, $t_{1}(598)=0.50, p=.62 ; t_{2}(38)=0.34, p=.74 .^{9}$ The perceived expertise of the experts was also the same in both cultures, $M_{\text {Dutch }}=3.29, S D=0.64$; $M_{\text {French }}=3.22, S D=0.74 ; t(598)=1.36, p=.17$.

The interaction between culture and evidence type suggests that there is a cultural difference between the French and the Dutch with respect to their susceptibility to different evidence types. To be able to better understand this cross-cultural difference, each participant's Preference for Numerical Information, Preference for Expert Information, and Need for Cognition were assessed. If scores on scales are to be compared directly, Van de Vijver and Leung (1997, pp. 88-89) strongly advise researchers to check whether participants with different cultural backgrounds used the scales in the same way. By employing Bachman and O'Malley's (1984) index, French participants were shown to use extreme scores more frequently than Dutch participants ( $p s<.001$ ). Therefore, the scores of all participants were standardized. A MANOVA using the standardized scores on the Preference for Numerical Information, the Preference for Expert Information, and the Need for Cognition scale did not reveal a significant difference between the French and the Dutch participants (Wilks' $\lambda=.997), F(3,595)=0.57, p=.64 .^{10}$

\section{Conclusion and Discussion}

Study 1 showed that there are cultural differences in the susceptibility of Dutch and French participants to anecdotal, statistical, causal, and expert evidence (RQ1). The pattern of results for the Dutch participants replicates that of a previous study in which anecdotal evidence proved to be the least persuasive and statistical evidence the most persuasive type of evidence (Hoeken \& Hustinx, 2003). For the French, statistical evidence did not prove to be more convincing than expert or causal evidence. Surprisingly, these differences in susceptibility to different evidence types were not accompanied by differences in preferences for different types of information or in Need for Cognition.

H1 predicted that expert evidence would be more persuasive for the French participants compared to the Dutch participants. This hypothesis was not supported. Given the adequate statistical power (see Note 9), the absence of an effect appears to reflect a genuine null-effect. The finding that expert evidence was as persuasive for the Dutch as it was for the French may be explained by the fact that high quality expert evidence was used. In the construction of the instantiations of the expert evidence, care was taken that they were normatively strong. Furthermore, comparisons revealed that the experts used were considered to be equally knowledgeable by the French and the Dutch participants.

If the French participants were more susceptible to expert evidence because it is provided by an expert, the fact that the expert is knowledgeable in a relevant field 
may have been less important to them than it was to the Dutch participants. A similar claim is made by the French communication scholar Breton (2003, p. 64), who argues that experts can influence people's opinions about an issue that is far from their own field of expertise. In order to test this assumption, the relevance of the expert's field to the claim at hand has to be manipulated. If being an expert is convincing for the French regardless of the specific claim, manipulation of the relevance of the field of expertise should result in an effect for the Dutch participants but not for the French participants. In order to assess whether the reduced susceptibility of the French participants for strong and weak expert evidence is restricted to expert evidence only, this effect has to be compared to the effect of evidence quality for another type of evidence. Sample size is a clear criterion for distinguishing strong statistical evidence from weak statistical evidence. Therefore, strong and weak statistical evidence was employed to serve as a comparison for the responses of French and Dutch participants to strong and weak expert evidence. If the French are susceptible to expert evidence regardless of its quality, even though they are capable of distinguishing between strong and weak statistical evidence, a three-way interaction between culture, evidence type, and evidence quality should arise. This prediction was tested in Study 2.

\section{Study 2}

\section{Method}

Material. Participants received 10 claims from Study 1 with manipulated evidence: 2 with normatively strong expert evidence, 2 with normatively weak expert evidence, 2 with normatively strong statistical evidence, 2 with normatively weak statistical evidence, and 2 without evidence. The other 10 claims from Study 1 were used as fillers (5 with anecdotal, and 5 with causal evidence). Normatively weak expert evidence was created by changing the relevant field of expertise into an irrelevant field of expertise. For statistical evidence, two sets of normatively strong and normatively weak statistical evidence were created by manipulating both the percentage presented and the sample size on which that percentage was based: " $78 \%$ of 314 persons" and " $74 \%$ of 381 persons" for the strong instantiations, and " $35 \%$ of 46 persons" and " $38 \%$ of 53 persons" for the weak instantiations.

Participants. The Dutch participants were students from universities in Amsterdam ( $n=73$; five classes), Delft $(n=21$; two classes), Enschede $(n=28$; three classes), Nijmegen $(n=77)$, and Tilburg $(n=101$; three classes $)$. The French participants studied in Besançon $(n=49)$, Paris $(n=56$; two classes), Roubaix $(n=58)$, Strasbourg ( $n=65$; six classes), and Tours $(n=72)$. More than $90 \%$ of the Dutch and French participants studied at the faculty of Humanities. There were more female participants in the French sample $(81.3 \%)$ than in the Dutch sample $(70.0 \%)$, $\chi^{2}(1)=10.32, p<.01 .^{11}$ The Dutch participants were slightly older $(M=20.64, S D=$ 1.91 , range 17-26) than the French participants $(M=20.19, S D=1.81$, range 17-30), $t(596.21)=2.97, p<.01$. This difference did not affect the persuasiveness of evidence, 
as age did not interact with evidence type, $F(1,598)=1.35, p=.25$, or evidence quality $(F[1,598]<1)$.

Instrumentation. The instrumentation was the same as in Study 1 except for two changes. First, next to the NFC scale (Dutch: $\alpha=.72$; French: $\alpha=.78$ ), and the PEI scale (Dutch: $\alpha=.75$; French: $\alpha=.79$ ), the Right-Wing Authoritarianism scale (RWA; Altemeyer, 1988) was included. The reliability of its 10 items ${ }^{12}$ proved to be adequate for the French participants $(\alpha=.71)$, but only marginally so for the Dutch participants $(\alpha=.60)$. Deleting items on the basis of factor analysis did not improve reliability. As the Dutch and French standardized scores on all three scales did not prove to differ $(p s>.10)$, they will not be discussed further in the results section.

The second change was the inclusion of a control question about the perceived quality of statistical evidence, in addition to the existing questions about the perceived expertise of the experts. The participants indicated which of two examples they would choose as proof for the generality of the occurrence of an effect on a 5point semantic differential with, on the left-hand side, "the effect occurs in 35\% of 46 persons" and, on the right-hand side, "the effect occurs in 78\% of 314 persons."

Design. All the participants received the 20 claims in exactly the same order in each of the five versions. The distribution of the manipulated types of evidence over the 10 experimental claims and the five versions, however, followed a balanced Latin square.

Procedure and statistical tests. The procedure followed was the same as for Study 1. The difference scores computed on the basis of the average scores on the claims without any evidence were analyzed through a 2 (culture) $\times 2$ (evidence quality) $\times$ 2 (evidence type) analysis of variance with culture as a between-subjects factor, and evidence type and evidence quality as within-subjects factors. To assess whether there were carry over effects, it was tested whether there were interaction effects between evidence type and evidence quality and the scores on the first items and those on the last items. The interactions were not significant $(p s>.11)$.

\section{Results}

Table 3 shows the persuasiveness of the strong and the weak instantiations of statistical and expert evidence for the Dutch and the French participants. The predicted three-way interaction between culture, evidence type, and evidence quality

Table 3 Persuasiveness of Evidence Types and Evidence Quality

\begin{tabular}{lccccc}
\hline & \multicolumn{2}{c}{ Dutch $(n=300)$} & & \multicolumn{2}{c}{ French $(n=300)$} \\
\cline { 2 - 3 } \cline { 5 - 6 } Evidence Type & $M$ & $S D$ & & $M$ & $S D$ \\
\hline Statistical & & & & & \\
$\quad$ Strong & 0.50 & 0.85 & & 0.25 & 0.91 \\
$\quad$ Weak & 0.19 & 0.86 & & 0.20 & 0.82 \\
$\quad$ Expert & & & & & \\
$\quad$ Strong & 0.35 & 0.88 & & 0.14 & 0.83 \\
$\quad$ Weak & 0.12 & 0.85 & & 0.19 & 0.83 \\
\hline
\end{tabular}


did not arise, $F_{1}(1,598)<1, F_{2}(1,9)<1$. There was a main effect of culture in the analysis by participants, $F_{1}(1,598)=5.37, p<.05, \eta^{2}=.01$, indicating that evidence was more effective for the Dutch participants $(M=0.29, S D=0.52)$ than for the French participants $(M=0.19, S D=0.56)$. However, this effect was not significant in the analysis by stimuli, $F_{2}(1,9)=2.19, p=.17$. There were also main effects of evidence type (statistical evidence: $M=0.28, S D=0.65$; expert evidence: $0.20, S D=$ $0.66), F_{1}(1,598)=7.91, p<.01, \eta^{2}=.01 ; F_{2}(1,9)=3.98, p=.08$, and of evidence quality (strong evidence: $M=0.31, S D=0.70$; weak evidence: $M=0.18, S D=0.65$ ), $F_{1}(1,598)=16.64, \quad p<.001, \eta^{2}=.03 ; \quad F_{2}(1,9)=8.71, \quad p<.05, \eta^{2}=.49$. The interactions between culture and evidence type, $F_{1}(1,598)=1.04, p=.31 ; F_{2}(1$, $9)=1.09, p=.32$, and between evidence type and evidence quality, $F_{1}(1,598)=2.23$, $p=.14 ; F_{2}(1,9)=1.57, p=.24$, were not significant.

However, there was a strong culture by evidence quality interaction: $F_{1}(1,598)=$ 16.02, $p<.001, \eta^{2}=.03 ; F_{2}(1,9)=26.13, p<.01, \eta^{2}=.74$. A further inspection of this interaction revealed a significant effect of culture for strong evidence (Dutch: $M=$ $0.42, S D=0.84$; French: $M=0.19, S D=0.90), F_{1}(1,598)=17.01, p<.001, \eta^{2}=.03$; $F_{2}(1,9)=5.38, p<.05, \eta^{2}=.37$, which was absent for the weak evidence (Dutch: $M=$ $0.16, S D=0.83$; French: $M=0.19, S D=0.86), F_{1}(1,598)<1 ; F_{2}(1,9)<1$. Apparently, there was a cultural difference for strong evidence, but not for weak evidence.

The above results suggested that the manipulation of argument quality has not been successful for the French participants. To assess whether this was the case, the manipulation check items for the expert evidence and the statistical evidence were analyzed. For expert evidence, there was a significant interaction between culture and evidence quality, $F(1,598)=43.93, p<.001, \eta^{2}=07$. Separate analyses for the Dutch and French participants revealed an effect of expert evidence quality on perceived expertise for both cultures, but the effect was much larger for the Dutch participants (strong evidence: $M=3.30, S D=0.83$; weak evidence: $M=2.32, S D=0.85), F_{1}(1$, $299)=255.81, p<.001, \eta^{2}=.46$, than for the French participants (strong evidence: $M=3.02, S D=0.86$; weak evidence: $M=2.61, S D=0.92), F_{1}(1,299)=46.48, p<$ $.001, \eta^{2}=.14$.

The perception of the quality of the strong and weak statistical evidence was measured by having participants choose which of two statistical evidence instantiations they regarded as stronger on a 5-point semantic differential (with 1 implying the supposedly weak statistical evidence and 5 implying the supposedly strong statistical evidence). For both the French and Dutch participants, the average score lay significantly above the neutral midpoint of the scale: French $(M=3.71, S D=$ $1.28), t(290)=9.52, p<.001$; Dutch $(M=4.39, S D=1.00), t(298)=24.12, p<.001$. However, as was the case for the expert evidence, the Dutch participants made a stronger distinction between the strong and the weak evidence than the French participants, $t(547.15)=7.12, p<.001$.

\section{Conclusion and Discussion}

Study 2 tested whether Dutch participants would be more persuaded by strong evidence than by weak evidence regardless of it being statistical evidence or expert 
evidence, and whether French participants would be more persuaded by strong statistical evidence than by weak statistical evidence, whereas evidence quality would not play a part in their susceptibility to expert evidence. These predictions were partly confirmed. The Dutch were persuaded by strong evidence more than by weak evidence regardless of evidence type, and the French were as susceptible to strong as to weak expert evidence. However, the predicted three-way interaction did not arise because the French participants were equally susceptible to strong as to weak statistical evidence. The absence of this three-way interaction effect, however, could not be attributed to the French participants' inability to distinguish between strong and weak evidence. Manipulation checks revealed that they preferred the strong statistical evidence to the weak statistical evidence and that they considered the strong experts as more knowledgeable on the issue than the weak experts (although these effects were both considerably weaker than for the Dutch participants).

As in Study 1, there were no differences between French and Dutch participants with respect to their Preference for Expert Information or Need for Cognition. RightWing Authoritarianism had been chosen as an additional instrument to detect cultural differences between Dutch and French participants with respect to their susceptibility to authority. However, no differences between the participants from these two cultures were obtained on this scale. The differences between the French and Dutch participants on the susceptibility to evidence types and evidence quality therefore cannot be explained by differences on these characteristics. We will return to this issue in the General Discussion.

\section{General Conclusion and Discussion}

This research responded to the various calls for empirical research on the impact of culture on the persuasiveness of evidence types. The susceptibility to different evidence types in two countries, France and The Netherlands, was compared in two studies. In both studies, cultural differences in susceptibility were found. In the first study, (strong) statistical evidence had more impact on the acceptance of claims for the Dutch participants than for the French. This result was also found in the second study, but only for strong statistical evidence; in the case of weak statistical evidence, Dutch participants were as unwilling as French participants to accept the claim. A similar effect was obtained for strong and weak expert evidence: Strong expert evidence had a larger impact for the Dutch participants than for the French participants, whereas weak expert evidence did not have much of an effect for either the Dutch or the French participants.

The reason for comparing France and The Netherlands was that we had expected cultural differences with respect to the impact of expert evidence. This expectation was based upon sociological research documenting differences with respect to power distance, that is, the extent to which differences in power are expected and accepted (Hofstede, 1980, 2001; Koopman et al., 1999). Studies in education have also revealed that teachers (i.e., experts) are considered omniscient in France (Blom, 1995; Planel, 1997). Furthermore, content-analytic studies did show differences in the use of 
experts in different cultures in commercial advertising (De Mooij, 1998, pp. 278-279) and public information leaflets (Hornikx et al., 2003), which were explained in terms of differences in power distance in different cultures. Finally, Pornpitakpan and Francis (2001) used differences in power distance to explain the fact that Thai participants were more convinced by source qualification than Canadian participants.

In Studies 1 and 2, personality characteristics were measured that could be argued to be related to power distance and that could be related to the susceptibility to expert evidence (Studies 1 and 2: Preference for Expert Information; Study 2: Right-Wing Authoritarianism). In both studies, and for both personality characteristics, no differences between the French and the Dutch participants were found. The absence of a difference may suggest that a cultural difference between France and The Netherlands with respect to power distance does not exist, or more accurately, that if it does exist, it does not translate itself into differences with respect to these personality characteristics. A similar absence of differences in personality characteristics was obtained in two other persuasion studies on cultural differences within Western Europe (Hoeken, Starren, Nickerson, Crijns, \& van de Brandt, 2007; Hoeken et al., 2003). Based upon the work by Hofstede (2001), cultural differences were expected in these studies on dimensions such as high-low uncertainty avoidance and masculinity-femininity. For each participant, his or her value hierarchy was measured to assess whether cultural differences were reflected in individual participants' value hierarchies. In both studies, no cultural differences in value hierarchies were found for participants from Belgium, England, France, Germany, The Netherlands, and Spain. Although outside the scope of this research, these results raise questions about the ways in which cultural differences documented at the level of groups can be detected at the level of individual participants.

Despite the absence of differences on several personality characteristics in the present research, cultural differences were nevertheless found in the persuasiveness of evidence types between the French and the Dutch participants. The Dutch participants were more susceptible to statistical and expert evidence than the French (Study 2). It may be concluded from this finding that the Dutch are easier to persuade than the French. However, the higher susceptibility of the Dutch participants to these types of evidence was limited to the cases in which the statistical evidence and the expert evidence were normatively strong; in the case of weak evidence, the Dutch were as reluctant to accept the claim as the French participants. The extent to which the latter were willing to accept a claim did not depend on the quality of the evidence in support of that claim.

The fact that the French participants' acceptance of a claim did not depend on the quality of the evidence may suggest that they did not pay attention to the evidence at all. In Study 1, however, which was very similar in method and sampling as Study 2, the French participants proved to be sensitive to differences in evidence quality: They were less susceptible to the (normatively weak) anecdotal evidence than the other types of evidence. They were also able to spot the manipulation of evidence quality in Study 2: When asked to choose between the strong and weak statistical evidence in support of a claim, they chose the statistical evidence with the higher sample size. 
They also considered the expert in the strong expert evidence condition to be more knowledgeable than in the weak expert evidence condition. However, the difference in perception between the strong and weak evidence manipulations was less pronounced for the French than for the Dutch. This raises the question as to whether the criteria used by Dutch and French participants to evaluate the quality of evidence are the same and/or carry the same weight. In future research, it would be interesting to chart what criteria people from different cultures use to assess the quality of evidence.

There are several limitations to the current research. First of all, the French and Dutch student samples were not representative of the French and Dutch populations. However, the sampling of participants at various universities and in various regions of the two countries made the two samples comparable. Second, the material consisted of short texts on relatively neutral issues. Whether similar results would be obtained with longer and more complicated texts on issues that participants were more involved in remains to be seen. The relatively large number of claims and evidence combinations participants were asked to respond to, however, is a major step forward from studies in which the acceptance of only one claim was studied. Third, although the manipulation of the different types of evidence focused on the content of the evidence, such a manipulation inevitably had consequences for the stylistic features of the evidence. The acceptance of a claim may have depended on such characteristics. One of the most plausible features in this respect would be evidence length, as people are known to sometimes employ the "length is strength" heuristic. If this evidence feature had had such an effect, however, any type of evidence would have led to a stronger acceptance of a claim regardless of its content. However, the results in Study 1 show that the inclusion of anecdotal evidence did not have an additional effect on the claims' acceptance for either the Dutch or the French participants, despite a considerable increase in evidence length. Furthermore, the analyses do not seem to warrant the suggestion that the acceptance of claims was influenced by evidence features such as length (see Note 5). A final limitation is that the responses of participants from only two countries were compared. More countries have to be included in future research in order to draw more general conclusions with respect to cultural differences in the persuasiveness of evidence types between Western cultures.

Despite the limitations discussed above, we believe that the studies reported here contribute to our understanding of cultural differences in persuasion processes. Most of such research has focused on comparing Asian and Western cultures. The differences found have been explained on the basis of differences in Confucian versus Aristotelian heritage. The experiments described in this paper have shown that differences can be found even among the heirs of Aristotle, because France and The Netherlands seem to differ in their susceptibility to different types of evidence and to evidence quality variations, despite their shared Grecian roots. The results of these experiments raise questions about the cultural relativity of norms used to distinguish strong from weak evidence, questions that should be addressed in future research. The results also show that answering the call for research on cultural differences in the relative persuasiveness of evidence types is an intriguing and fruitful enterprise. 


\section{Notes}

[1] As pointed out by anonymous reviewers of this manuscript, there is a relation between evidence type and argument type. For instance, expert evidence is typically used in an argument by authority and statistical evidence in an argument by generalization. From that perspective, this study could have been framed as a study on types of arguments. However, we chose to take the perspective of different types of evidence because the anecdotal evidence and the statistical evidence would both be considered arguments by generalization. In our study, we used claims about general principles that were either supported by one example (anecdotal evidence) or by a numerical summary of a number of cases (statistical evidence). Both can serve as evidence in support of an argument by generalization.

[2] Reynolds and Reynolds' (2002) definition of evidence is not the only definition of evidence. McCroskey reserves the label evidence for "opinions of others or facts attested to by others" (1986, p. 101). If this definition is used, expert evidence is evidence, but anecdotal and causal evidence are not. With respect to statistics presented as evidence, they supposedly result from a study, which suggests that the data were not collected by the source propagating the claim, but by an unnamed researcher. As a result, these data can be regarded as evidence from an unidentified source (see Reinard, 1991, for a thorough discussion of these issues). A fundamental discussion on the merits and problems of the different definitions and operationalizations of evidence is a much needed one, but it falls outside the scope of this paper.

[3] For European countries, large power distance correlates positively and significantly with high uncertainty avoidance $(r=.78$; Hofstede, 2001, p. 150). As a consequence, France and The Netherlands also differ on the cultural dimension of uncertainty avoidance, "The extent to which the members of a culture feel threatened by uncertain or unknown situations." This dimension, however, does not seem to relate to a specific type of evidence.

[4] The following templates were used. For anecdotal evidence: "Since [first name + last name] from [place] experienced the cause, the consequence has taken place." For statistical evidence: "A [Dutch/French] study among $N$ [Dutch/French] participants showed that the consequence occurred with $\mathrm{X} \%$ of them as a result of the cause" or "The results of a [Dutch/ French] study among $N$ [Dutch/French] participants showed that the consequence occurred with X\% of them as a result of the cause." For expert evidence: "According to Prof. Dr [name] from the university of [place], an expert/a specialist in the field of [research area], [the claim]" or "Prof. Dr [name], an expert/a specialist in the field of [research area] at the university of [place], underscores [the claim]." For causal evidence: "[The cause in the claim] leads to [a specific effect] / Because of [the cause in the claim] [a specific effect occurs], which produces [the effect mentioned in the claim]."

[5] As one of the reviewers remarked, the evidence instantiations not only differ in type (e.g., anecdotal, expert), but may also differ in length, readability, concreteness, and identification of sources. One signal of such confounding would be the presence of heterogeneous variances between evidence types without the presence of a ceiling or floor effect (see Reinard, 2006, for an interesting discussion of this issue). Mauchly's sphericity tests for all main effects in the repeated measures designs in Studies 1 and 2 were not significant. However, the absence of a significant effect does not guarantee that there was no such effect. We conducted an analysis to check for the effect of length. The length of the instantiations of the four types of evidence was computed, and a main effect of evidence type on evidence length occurred: France, $F(3,76)=36.52, p<.001, \eta^{2}=.59 ;$ Netherlands, $F(3,76)=27.64, p<.001, \eta^{2}=.52$. Subsequently, ANCOVAs with length as covariate showed that for the Dutch material, evidence length did not affect the relative persuasiveness of evidence types, $F(1,75)<1$, and the main effect of type of evidence was still present, $F(3,75)=4.70, p<.01, \eta^{2}=.16$. For the French material, there was no effect of length as covariate, $F(1,75)<1$, but there was no longer a main effect of type of evidence on persuasiveness, $F(3,75)=1.76, p=.16$. Therefore, 
evidence length seems to have played a role for the French participants, but - in the absence of an effect for length - it is not a strong alternative explanation for the difference in persuasiveness of the types of evidence. In Study 2, main effects of evidence type on evidence length were absent (both cultures, $F[3,36]<1$ ), implying that evidence length did not contribute to evidence type effects.

[6] Although we realize that using a single item to measure probability lowers the reliability of the measurement, we still chose for a single item measurement. In a pretest of a similar questionnaire in a previous study in which three semantic differentials were used to measure probability (Hoeken \& Hustinx, 2003), participants expressed their irritation about having to rate their opinion on three almost identical differentials twenty times. As the scale reliability of the three semantic differentials was good $(\alpha=.89)$, one of them was selected: very improbable-very probable.

[7] The six items were: (1) If an expert says it is the case, then it is the case; (2) Judgments of experts are very important to me; (3) I prefer to base my decisions on the opinion of an expert; (4) The judgment of an expert needs to be believed; (5) I accept that there are people who know more about a topic than me; (6) Knowledge makes experts powerful.

[8] Claim 2: $M_{\text {Dutch }}=3.44, S D=0.99 ; M_{\text {French }}=2.88, S D=0.98 ; t(117)=3.12, p<.01$; Claim 19: $M_{\text {Dutch }}=3.10, S D=1.33 ; M_{\text {French }}=2.59, S D=1.05 ; t(113.73)=2.32, p<.05$; Claim 12: $M_{\text {Dutch }}=3.07, S D=1.28 ; M_{\text {French }}=3.75, S D=1.08 ; t(115.87)=3.16, p<.01$.

[9] Statistical power to detect a hypothesized effect was .99 for large $(d=.80)$ and medium effect sizes $(d=.50)$, and .97 for a small effect size $(d=.20$; Cohen, 1988).

[10] Most correlations between these three scales and the different types of evidence were not significant in both samples; the same applies to Study 2. Significant correlations ranged between .12 and .24 .

[11] Participants' sex did not affect the relative persuasiveness of the evidence types $(F[1,597]<1)$, but it did affect the relative persuasiveness of evidence quality, $F(1,597)=4.71, p<.05$, $\eta^{2}=.01$. In fact, strong evidence was more persuasive to male than to female participants, $t(597)=2.25, p<.05$. However, more importantly, for both male, $t(145)=3.41, p<.01$, and female participants, $t(452)=2.55, p<.05$, strong evidence was more persuasive than weak evidence.

[12] According to nine pairs of Dutch students and the first author, items 2, 3, 5, 7, 12, 17, 22, 23, 26, and 29 from Altemeyer (1988, pp. 22-23) were related most strongly to power distance.

\section{References}

Allen, M., \& Preiss, R. W. (1997). Comparing the persuasiveness of narrative and statistical evidence using meta-analysis. Communication Research Reports, 14, 125-131.

Altemeyer, B. (1988). Enemies of freedom: Understanding right-wing authoritarianism. San Francisco: Jossey-Bass.

Areni, C. S., \& Lutz, R. J. (1988). The role of argument quality in the Elaboration Likelihood Model. In M. J. Houston (Ed.), Advances in consumer research (Vol. 15, pp. 197-203). Provo, UT: Association for Consumer Research.

Bachman, J. G., \& O'Malley, P. M. (1984). Yea-saying, nay-saying, and going to extreme: Blackwhite differences in response styles. Public Opinion Quarterly, 48, 491-509.

Blom, S. (1995). Intellectuele vorming in Nederland en Frankrijk: Een vergelijkend onderzoek naar deelname aan intellectuele vorming in het Franse en Nederlandse voortgezet onderwijs [Intellectual formation in The Netherlands and France: A comparative study of the participation in intellectual formation in Dutch and French secondary education]. Groningen, The Netherlands: Wolters-Noordhoff.

Breton, P. (2003). L'argumentation dans la communication [Argumentation in communication] (3rd ed.). Paris: La Découverte. 
Brislin, R. W. (1980). Translation and content analysis of oral and written material. In H. C. Triandis, \& J. W. Berry (Eds.), Handbook of cross-cultural psychology: Methodology (pp. 389444). Boston: Allyn \& Bacon.

Cacioppo, J. T., Petty, R. E., \& Kao, C. F. (1984). The efficient assessment of need for cognition. Journal of Personality Assessment, 48, 306-307.

Chaiken, S. (1987). The heuristic model of persuasion. In M. P. Zanna, J. M. Olson, \& C. P. Herman (Eds.), Social influence: The Ontario symposium (Vol. 5, pp. 3-39). Hillsdale, NJ: Lawrence Erlbaum Associates, Inc.

Cohen, J. (1988). Statistical power analysis for the behavioral sciences (2nd ed.). Hillsdale, NJ: Lawrence Erlbaum Associates, Inc.

De Mooij, M. (1998). Global marketing and advertising. Thousand Oaks, CA: Sage.

Edwards, K., \& Smith, E. E. (1996). A disconfirmation bias in the evaluation of arguments. Journal of Personality and Social Psychology, 71, 5-24.

Elmes, D. G., Kantowitz, B. H., \& Roediger, H. L. (1992). Research methods in psychology (3rd ed.). St Paul, MN: West.

Feteris, E. T. (2002). Pragmatic argumentation in a legal context. In F. H. van Eemeren (Ed.), Advances in pragma-dialectics (pp. 243-260). Amsterdam: Sic Sat/Newport News, VA: Vale Press.

Greene, K., \& Brinn, L. S. (2003). Messages influencing college women's tanning bed use: Statistical versus narrative evidence format and a self-assessment to increase perceived susceptibility. Journal of Health Communication, 8, 443-461.

Harkness, J. A., van de Vijver, F. J. R., \& Mohler, P. P. (2003). Cross-cultural survey methods. Hoboken, NJ: Wiley.

Hesslow, G. (1988). The problem of causal selection. In D. J. Hilton (Ed.), Contemporary science and natural explanation: Commonsense conceptions of causality (pp. 11-32). Brighton, UK: Harvester Press.

Hoeken, H., van den Brandt, C., Crijns, R., Domínguez, N., Hendriks, B., Planken, B., et al. (2003). International advertising in Western Europe: Should differences in uncertainty avoidance be considered when advertising in Belgium, France, The Netherlands and Spain? Journal of Business Communication, 40, 195-218.

Hoeken, H., \& Hustinx, L. (2003). The relative persuasiveness of different types of evidence. In F. H. van Eemeren, J. A. Blair, C. A. Willard, \& A. F. Snoeck Henkemans (Eds.), Proceedings of the fifth conference of the International Society for the Study of Argumentation (pp. 497-501). Amsterdam: Sic Sat.

Hoeken, H., Starren, M., Nickerson, C., Crijns, R., \& van de Brandt, C. (2007). Is it necessary to adapt advertising appeals for national audiences in Western Europe? Journal of Marketing Communications, 13, 19-38.

Hofstede, G. (1980). Culture's consequences: International differences in work-related values. Beverly Hills, CA: Sage.

Hofstede, G. (2001). Culture's consequences: Comparing values, behaviors, institutions, and organizations across nations (2nd ed.). Thousand Oaks, CA: Sage.

Hornikx, J., Starren, M., \& Hoeken, H. (2003). Cultural influence on the relative occurrence of evidence types. In F. H. van Eemeren, J. A. Blair, C. A. Willard, \& A. F. Snoeck Henkemans (Eds.), Proceedings of the fifth conference of the International Society for the Study of Argumentation (pp. 531-536). Amsterdam: Sic Sat.

Jung, J. M., \& Kellaris, J. J. (2006). Responsiveness to authority appeals among young French and American consumers. Journal of Business Research, 59, 735-744.

Koopman, P. L., Den Hartog, D. N., Konrad, E., Akerblom, S., Audia, G., Bakacsi, G., et al. (1999). National culture and leadership profiles in Europe: Some results from the GLOBE study. European Journal of Work and Organizational Psychology, 8, 503-520. 
Kruglanski, A. W., Raviv, A., Bar-Tal, D., Raviv, A., Sharvit, K., Ellis, S., et al. (2005). Says who? Epistemic authority effects in social judgment. Advances in Experimental Social Psychology, 37, 345-392.

McCroskey, J. C. (1969). A summary of experimental research on the effects of evidence in persuasive communication. Quarterly Journal of Speech, 55, 169-176.

McCroskey, J. C. (1986). An introduction to rhetorical communication (5th ed.). Englewood Cliffs, NJ: Prentice Hall.

Nisbett, R. E. (2003). The geography of thought: How Asians and Westerners think differently ... and why. London: Nicolas Brealey.

Nisbett, R. E., Peng, K., Choi, I., \& Norenzayan, A. (2001). Culture and systems of thought: Holistic versus analytic cognition. Psychological Review, 108, 291-310.

Norenzayan, A., Smith, E. E., Kim, B. J., \& Nisbett, R. E. (2002). Cultural preferences for formal versus intuitive reasoning. Cognitive Science, 26, 653-684.

Park, H. S., Levine, T. R., Kingsley Westerman, C. Y., Orfgen, T., \& Foregger, S. (2007). The effect of argument quality and involvement type on attitude formation and attitude change: A test of dual-process and social judgment predictions. Human Communication Research, 33, 81-102.

Petty, R. E., \& Cacioppo, J. T. (1986). Communication and persuasion: Central and peripheral routes to attitude change. New York: Springer.

Planel, C. (1997). National cultural values and their role in learning: A comparative ethnographic study of state primary schooling in England and France. Comparative Education, 33, 349-373.

Pornpitakpan, C. (2004). The persuasiveness of source credibility: A critical review of five decades' evidence. Journal of Applied Social Psychology, 34, 243-281.

Pornpitakpan, C., \& Francis, J. N. P. (2001). The effect of cultural differences, source expertise, and argument strength on persuasion: An experiment with Canadians and Thais. Journal of International Consumer Marketing, 13, 77-101.

Reinard, J. C. (1988). The empirical study of the persuasive effects of evidence: The status after fifty years of research. Human Communication Research, 15, 3-59.

Reinard, J. C. (1991). Foundations of argument: Effective communication for critical thinking. Dubuque, IA: W. C. Brown.

Reinard, J. C. (2006). Communication research statistics. Thousand Oaks, CA: Sage.

Reynolds, R. A., \& Reynolds, J. L. (2002). Evidence. In J. P. Dillard \& M. Pfau (Eds.), The persuasion handbook: Developments in theory and practice (pp. 427-444). Thousand Oaks, CA: Sage.

Schellens, P. J., \& de Jong, M. (2004). Argumentation schemes in persuasive brochures. Argumentation, 18, 295-323.

Schellens, P. J., \& Verhoeven, G. (1994). Argument en tegenargument: Een inleiding in de analyse en beoordeling van betogende teksten [Argument and counterargument: An introduction to the analysis and evaluation of argumentative texts]. Groningen, The Netherlands: Martinus Nijhoff.

Toulmin, S. E. (1958). The uses of argument. Cambridge, UK: Cambridge University Press.

Van de Vijver, F. J. R., \& Leung, K. (1997). Methods and data analysis for cross-cultural research. Thousand Oaks, CA: Sage.

Van Enschot-Van Dijk, R., Hustinx, L., \& Hoeken, H. (2003). The concept of argument quality in the Elaboration Likelihood Model: A normative and empirical approach to Petty and Cacioppo's "strong" and "weak" arguments. In F. H. van Eemeren, J. A. Blair, C. A. Willard, \& A. F. Snoeck Henkemans (Eds.), Anyone who has a view: Theoretical contributions to the study of argumentation (pp. 319-335). Dordrecht, The Netherlands: Kluwer.

Viswanathan, M. (1993). The measurement of individual differences in preference for numerical information. Journal of Applied Psychology, 78, 741-752. 


\section{Appendix: The 20 Claims in Order of Appearance in Study 1, and Whether They} Were Used in Study 2

1. Being employed as an intermediary by an employment agency helps you to find a job later.

2. Compulsory driving lessons for people over 70 reduces their uncertainty in traffic.

3. Boys' performance at school can be improved by putting them next to girls in class. (2)

4. The consumption of basil in tomato pasta sauce improves sporting performance. (2)

5. Playing slow music in supermarkets increases sales.

6. Keeping photos of family and friends on your desk raises your productivity at work. (2)

7. A course of fruit juice helps people to attain their desired weight and maintain that weight.

8. People's fear of driving cars can be taken away if they ride along with a truck driver for a day. (2)

9. Playing party games helps young criminals to become more socialized. (2)

10. If art history students take a course that is oriented to the labor market, they will have better opportunities on that market.

11. Listening to classical music helps students to absorb a lot of knowledge in a short period of time. (2)

12. Salesmen feel more involved in their company when they use new media, such as a laptop with an Internet connection at home.

13. The consumption of cold beverages during exams leads to lower achievement in students.

14. Waiters who repeat customers' orders verbatim receive higher tips. (2)

15. If publicity agents watch cartoons that were popular in their youth, they will regain their creativity.

16. Wearing a tie too tightly leads to reduced sight. (2)

17. The introduction of an education protocol against bullying prevents children from falling victim to such behavior.

18. People's fear of flying can be decreased if they take part in a balloon flight. (2)

19. Regular job rotation within a company leads to higher productivity at work. (2)

20. Playing computer games has a positive impact on people's sense of direction. 